\title{
Systematic Review and Meta-analysis: Immunosuppressive Agents for the Treatment of Primary Biliary Cholangitis
}

\author{
Mengfan Du' ${ }^{1}$, Bing Xu' ${ }^{1 *}$, Ru Xiang ${ }^{1}$, Qian $\mathrm{Li}^{2}$, Yang Chen ${ }^{2}$, Yu Fan ${ }^{2}$ \\ ${ }^{1}$ The Medical Technology College of Shaanxi University of Chinese Medicine, Xianyang 712046, Shaanxi Province, China \\ ${ }^{2}$ The Basic Medical College of Shaanxi University of Chinese Medicine, Xianyang 712046, Shaanxi Province, China \\ *Corresponding author: Bing Xu, xubing-95@163.com
}

\begin{abstract}
Background and aim: There are still no clinically satisfactory therapy for PBC. This study was performed to assess the safety and efficacy of IAs for the therapy of PBC. Methods: Relevant studies were identified and selected by searching PubMed, Web of Science and Cochrane Library databases. The primary outcome was defined as the need for mortality or liver transplantation. Adverse effects and liver biochemical variables were a secondary outcome. Results: Nine randomized controlled trials, involving six different treatment regimens with a total of 996 patients, were included in the analysis. On meta-analysis, IAs was not associated with a reduction in risk of mortality or liver transplantation (risk ratio [RR]: $0.92,95 \%$ confidence interval [CI]: 0.69-1.22, $P=0.57, I^{2}=0 \%$, and have resulted in more adverse effects (RR: 1.44, $95 \%$ CI: $1.08-1.92, P=0.01, I^{2}=19 \%$ ). Subgroup analysis showed that IAs monotherapy caused adverse effects such as diarrhea, abdominal pain, and renal insufficiency (RR: $1.36,95 \% \mathrm{CI}: 1.01-1.82, P=0.04, I^{2}=48 \%$ ). IAs therapy did not prominently improve markers of liver function except for alkaline phosphatases (weighted mean difference [WMD]: -0.38 , 95\% CI: -0.62 to $-0.14, P=0.002$ ). Conclusions: IAs cannot reduce the risk of mortality or liver transplantation, whether in IAs monotherapy or combination therapy, and even be associated with more adverse effects.
\end{abstract}

Keywords: Primary biliary cholangitis; Immunosuppressive agents; Meta-analysis

Publication date: July 2021; Online publication: July 31, 2021

\section{Introduction}

Primary biliary cholangitis (PBC) is a prototypical autoimmune liver disease associated with multiple immune disorders ${ }^{[1]}$, and is characterized by inflammation and progressive destruction of the small and medium size intrahepatic bile ducts, leading to periportal inflammation, fibrosis, eventually progressing to cirrhosis and liver failure ${ }^{[2]}$. The prevalence of primary biliary cirrhosis ranges from 1.91 to $40 \cdot 20$ per 100000 people and has increased over time. PBC is mainly diagnosed in women, with a female-to-male sex ratio averaging approximately 10:1, and usually occurs in the $50 \mathrm{~s}$ and $60 \mathrm{~s}{ }^{[3]}$.

The pathogenesis of the disease is unknown, which is related to genetic factors, environment, microRNAs, immune dysregulation, cholestasis and other factors, and there is still no clinically satisfactory therapy. Currently, ursodeoxycholic acid (UDCA) is the only first-line drug approved by the Food and Drug Administration (FDA) for the treatment of PBC ${ }^{[4]}$. Several randomized trials and long-term observational studies have shown that UDCA improves serum liver biochemical indices, delays histological progression, the development of portal hypertension and its complications, and improves survival without transplantation ${ }^{[5,6]}$. Unfortunately, approximately $40 \%$ of patients display an incomplete response to UDCA and have a higher risk of developing cirrhosis, hepatocellular carcinoma, and 
end-stage liver disease that eventually leads to the need for liver transplantation or death from liver-related causes, indicating that additional therapeutic options are warranted [7,8].

In recent years, with the continuous development of immunological technology, immunosuppressive agents (IAs) such as colchicine, azathioprine, cyclosporine, prednisolone and Mycophenolate mofetil have made great progress in the treatment of autoimmune diseases ${ }^{[9,10]}$. and the combination therapy with IAs and UDCA is widely used ${ }^{[11]}$. Therefore, we performed this meta-analysis to determine the safety and efficacy of these interventions.

\section{Materials and methods}

\subsection{Search Strategy}

A comprehensive and systematic literature search was performed to identify all reports in PubMed, the Cochrane Library, Google scholar (updated to September 2020). The search terms included: keywords and/or medical subject heading (MeSH) for "primary biliary cholangitis (PBC)", "treatments, immunosuppressive agents" and "randomized controlled trial". Some additional search terms including "Glucocorticoids", "Rituximab", "Colchicine", "Cyclosporin", "Methotrexate", "Mycophenolate mofetil" were used to keep the result list complete.

\subsection{Inclusion Criteria:}

(1) Randomized controlled trials (RCTs) that compared IAs as monotherapy or combination therapy (which defined as IA co-administered with IA, IA co-administration therapy with UDCA) to placebo or UDCA or placebo plus UDCA in patients with PBC were included.

(2) From 1990 to September 2020.

(3) For different reports of the same research, studies with more complete outcome reports were selected.

(4) Studies should report mortality or liver transplantation.

(5) Irrespective of blinding, language, publication status.

\subsection{Risk of bias and quality assessment}

Quality of included studies was evaluated with the Cochrane risk of bias tools. Using this tool, studies were deemed to be high, low, or unclear risk of bias by a score of 0-6 (low risk=1, unclear and high risk=0).

\subsection{Data extraction}

Data were independently abstracted from each study by two authors (Meng-Fan Du and Ru Xiang), and any disagreement regarding data extraction was resolved by discussion between the authors.

\subsection{Statistical analysis}

Review Manager 5.3 provided by the Cochrane Collaboration was used to perform meta-analysis. For dichotomous outcomes, we calculated the risk ratio (RR), and for continuous outcomes, weighted mean difference (WMD), all with 95\% confidence intervals (CI).

\section{Results}

\subsection{Characteristics of included studies}

Out of 188 records that met the search criteria (Figure 1.), nine studies ${ }^{[12-20]}$ evaluated in our meta-analysis included a total of 996 patients (505 patients with monotherapy or combination therapy and 491 patients with placebo or UDCA). Four RCTs compared Methotrexate, Cyclosporin A, Rituximab 
with placebo. Three RCTs compared Colchicine plus UDCA with UDCA or placebo plus UDCA, two RCTs compared 
Methotrexate plus UDCA, UDCA plus prednisone or azathioprine with UDCA plus placebo. The mean age of the treatment group and control group was 53.1 and 54, and the majority of patients were female, accounting for $91.8 \%$ and $89.7 \%$ respectively. The duration of eligible trials varied from one year to ten years. The characteristics of the trials are listed in Table 1.

\subsection{Quality of Included Studies}

Of the nine RCTs reviewed, two were considered to be at a low risk of bias overall. Six trials reported the dropout numbers in the treatment (62 patients) and the control (61 patients) groups, three studies did not report the exact dropout rate. Intention-to-treat analyses were applied in 7 RCTs. (Figure 2.)

\subsection{Primary outcome: liver transplantation or mortality}

Nine trials, which included 996 patients, reported data regarding this end point (Figure 3.). 72 of 505 patients in the treatment groups and 76 of 491 patients in the control groups were reported dead or had liver transplantation. The results showed that IAs had no significant effect on mortality or liver transplantation (RR: $0.92,95 \% \mathrm{CI}: 0.69-1.22, P=0.57)$, and there was no significant heterogeneity $\left(I^{2}=\right.$ $0 \%$ ). The results of the subgroup analysis based on IAs monotherapy or combination therapy showed that the four monotherapy treatments had no significant difference on the mortality or the rate of liver transplantation (RR: 0.93, 95\% CI: 0.67-1.28, $P=0.66, I^{2}=32 \%$ ). Compared with control group, co-administration therapy had no significant difference in liver transplantation or mortality rates (RR: 0.90, 95\% CI: $0.50-1.61, P=0.73, I^{2}=0 \%$ ).

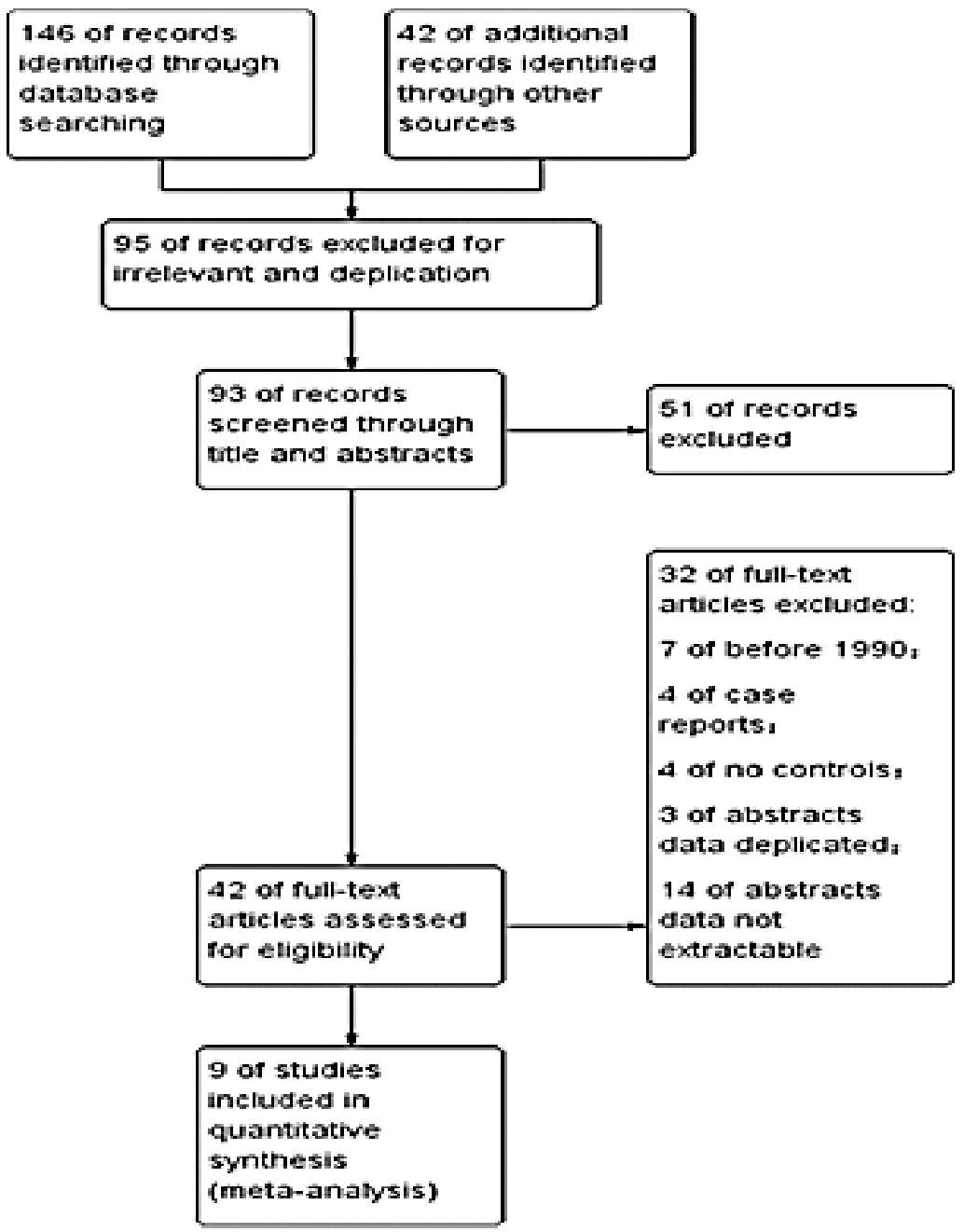

Figure 1. Flow diagram of studies identified, excluded, and included 
Table1. Characteristics of included studies.

\begin{tabular}{|c|c|c|c|c|c|c|c|c|}
\hline Study & $\begin{array}{l}\text { Treatment } \\
\text { options }\end{array}$ & $\begin{array}{c}\text { Peroid } \\
\text { (year) }\end{array}$ & $\begin{array}{l}\text { Age } \\
\text { mean }\end{array}$ & $\begin{array}{l}\text { Gender } \\
\text { (female) }\end{array}$ & $\begin{array}{l}\text { Sample } \\
\text { size (n) }\end{array}$ & $\begin{array}{c}\text { Total } \\
\text { (n) }\end{array}$ & $\begin{array}{c}\text { Primary } \\
\text { outcome } \\
\text { (n) } \\
\end{array}$ & $\begin{array}{c}\text { Adverse } \\
\text { effect } \\
\text { (n) }\end{array}$ \\
\hline \multirow{2}{*}{$\begin{array}{c}\text { Maek T } \\
1999\end{array}$} & MTX & \multirow{2}{*}{6} & 57 & $93 \%$ & 30 & \multirow{2}{*}{60} & 8 & 21 \\
\hline & Placebo & & 57 & $90 \%$ & 30 & & 6 & 19 \\
\hline \multirow{2}{*}{$\begin{array}{c}\text { Khanna } \\
2019\end{array}$} & Rituximab & \multirow{2}{*}{1} & 55.9 & $97 \%$ & 29 & \multirow{2}{*}{57} & 0 & 0 \\
\hline & Placebo & & 53.3 & $97 \%$ & 28 & & 0 & 4 \\
\hline \multirow{2}{*}{$\begin{array}{c}\text { Almasio } \\
2000\end{array}$} & $\begin{array}{l}\text { Colchicine + } \\
\text { UDCA }\end{array}$ & \multirow{2}{*}{3} & 53.3 & $89 \%$ & 46 & \multirow{2}{*}{90} & 2 & NR \\
\hline & $\begin{array}{l}\text { UDCA + } \\
\text { Placebo }\end{array}$ & & 55.5 & $91 \%$ & 44 & & 3 & NR \\
\hline \multirow{2}{*}{$\begin{array}{c}\text { Martin L } \\
1993\end{array}$} & $\begin{array}{c}\text { Cyclosporin } \\
\text { A }\end{array}$ & \multirow{2}{*}{4} & 53.9 & $86 \%$ & 176 & \multirow{2}{*}{349} & 44 & 34 \\
\hline & Placebo & & 54.2 & $85 \%$ & 173 & & 46 & 18 \\
\hline \multirow{2}{*}{$\begin{array}{c}\text { Frank HJ } \\
1998\end{array}$} & $\begin{array}{l}\text { UDCA + } \\
\text { Prednisone }\end{array}$ & \multirow[b]{2}{*}{1} & 52.7 & $89 \%$ & 26 & \multirow{2}{*}{50} & 1 & NR \\
\hline & $\begin{array}{l}\text { UDCA + } \\
\text { Placebo }\end{array}$ & & 52 & $92 \%$ & 24 & & 2 & NR \\
\hline \multirow{2}{*}{$\begin{array}{c}\text { Renee E } \\
1996\end{array}$} & $\begin{array}{c}\text { Colchicine }+ \\
\text { UDCA }\end{array}$ & \multirow[t]{2}{*}{2} & 55 & $89 \%$ & 37 & \multirow{2}{*}{74} & 2 & 1 \\
\hline & UDCA & & 52 & $81 \%$ & 37 & & 0 & 0 \\
\hline \multirow{2}{*}{$\begin{array}{c}\text { Burton C } \\
2005\end{array}$} & $\begin{array}{l}\text { UDCA + } \\
\text { MTX }\end{array}$ & \multirow{2}{*}{7.6} & $\begin{array}{c}50.3 / \\
50.4\end{array}$ & $\begin{array}{l}92 \% / \\
93 \%\end{array}$ & 132 & \multirow{2}{*}{265} & 14 & 4 \\
\hline & $\begin{array}{l}\text { UDCA + } \\
\text { Placebo }\end{array}$ & & $\begin{array}{c}50.3 / \\
54\end{array}$ & $\begin{array}{c}97 \% / \\
88 \%\end{array}$ & 133 & & 16 & 2 \\
\hline \multirow{2}{*}{$\begin{array}{c}\text { Takaaki I } \\
1996\end{array}$} & $\begin{array}{l}\text { Colchicine }+ \\
\text { UDCA }\end{array}$ & \multirow{2}{*}{4.5} & 57 & $90 \%$ & 10 & \multirow{2}{*}{22} & 0 & 2 \\
\hline & UDCA & & 64 & $83 \%$ & 12 & & 0 & 0 \\
\hline \multirow{2}{*}{$\begin{array}{c}\text { Russel HW } \\
1990\end{array}$} & Cyclosporine & 1 & 45.5 & $100 \%$ & 19 & \multirow{2}{*}{29} & 1 & 15 \\
\hline & Placebo & & 48 & $93 \%$ & 10 & & 3 & 5 \\
\hline
\end{tabular}




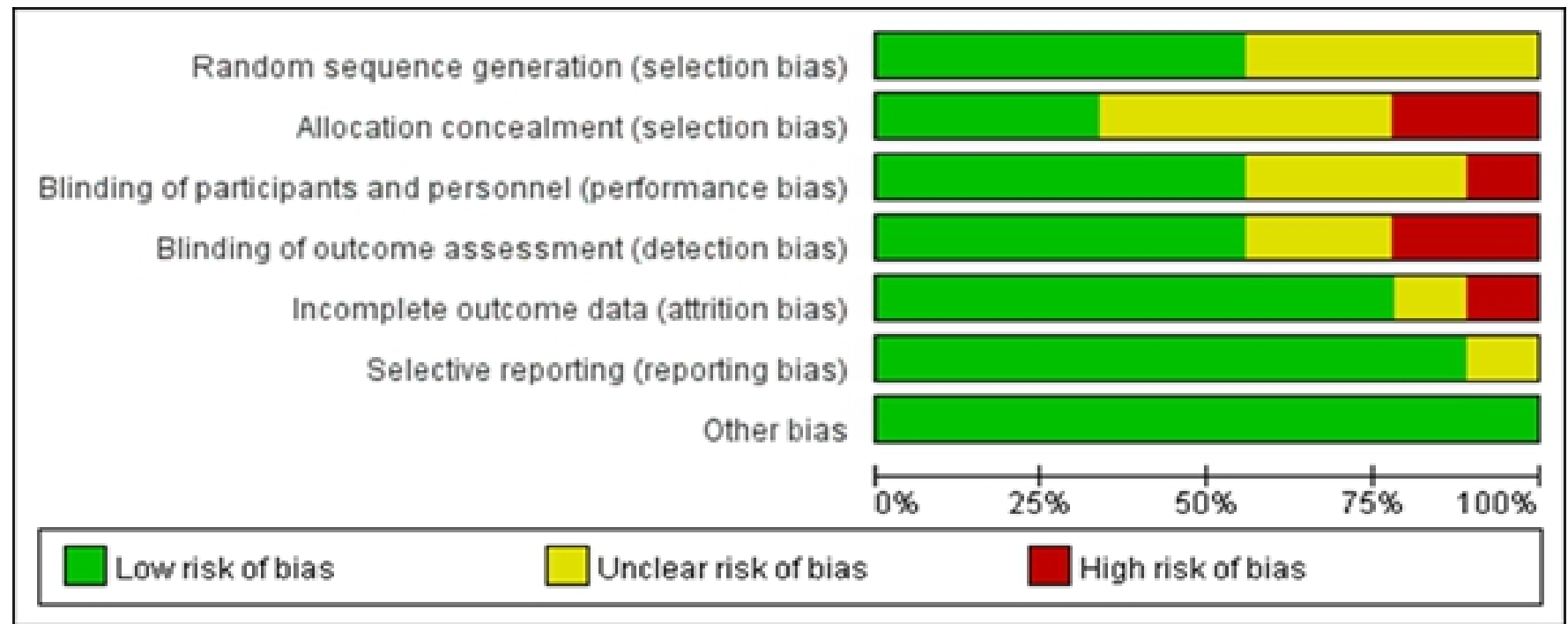

Figure 2. Risk of bias graph: review of authors' judgments regarding each risk of bias item presented as percentages across all included studies

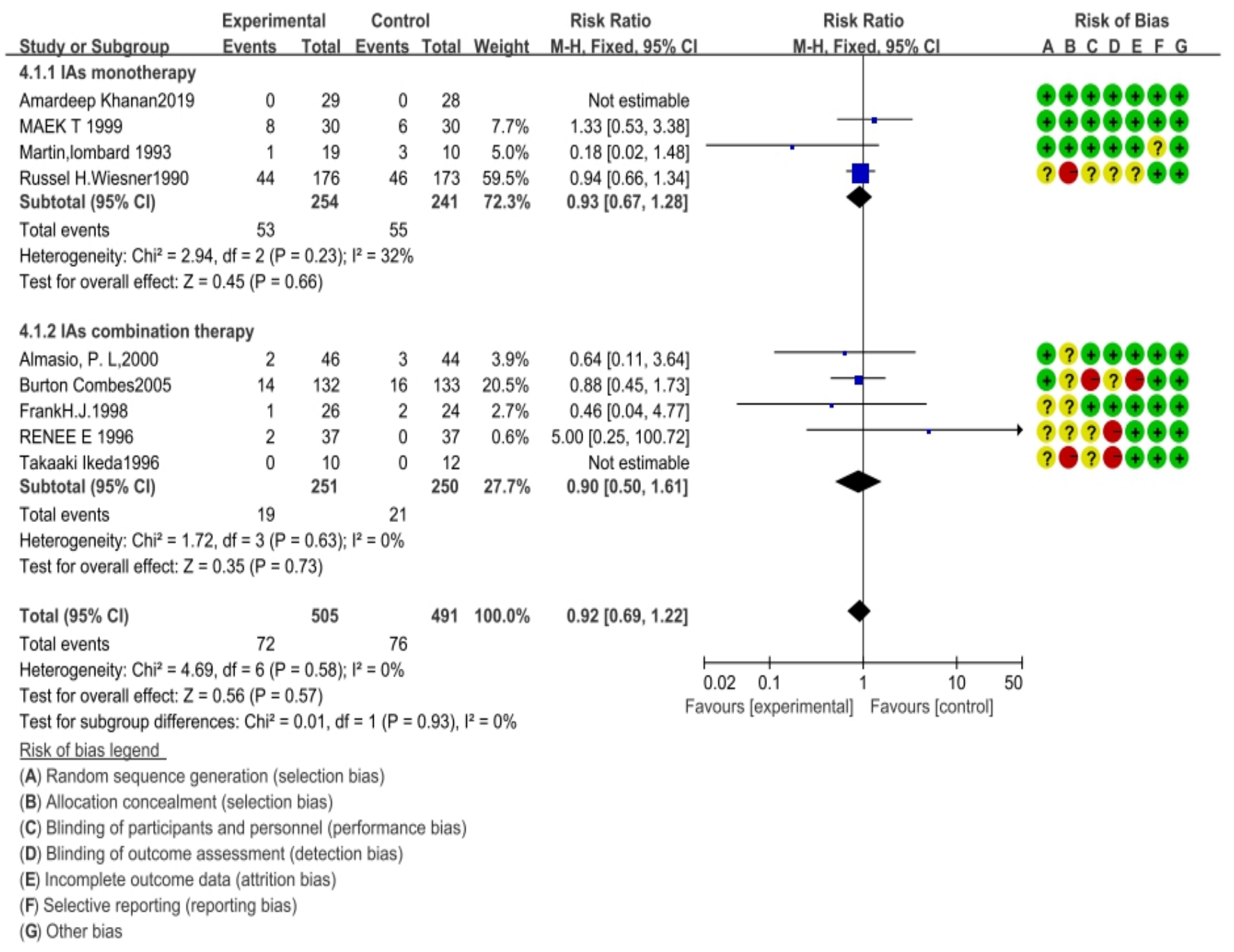

Figure 3. Forest plot of mortality or liver transplantation 


\subsection{Secondary outcome: adverse effects}

7 RCTs including 125 patients (77 patients in the treatment groups, 48 patients in the control groups) provided information on adverse effects and could be included in the analysis (Figure 4.). Meta-analysis showed that IAs therapy might cause more adverse effects (RR: $1.44,95 \% \mathrm{CI}: 1.08-1.92, P=0.01, I^{2}=$ $19 \%$ ). The subgroup analysis showed no significant difference in the rates of adverse effects for IAs co-administration therapy compared with UDCA or UDCA plus placebo (RR: $2.79,95 \%$ CI: 0.76-10.19, $P=0.12, I^{2}=0 \%$ ). Unfortunately, IAs monotherapy was associated with a significant increase in adverse effects (RR: $1.36,95 \% \mathrm{CI}: 1.01-1.82, P=0.04, I^{2}=48 \%$ ). Adverse effects mainly included fatigue, mouth ulcers, alopecia, marrow depression [16]; pneumonia, thrombocytopenia ${ }^{[18]}$; hirsutism, acne, menorrhagia, renal impairment, hypertension ${ }^{[19]}$; multiple peripheral neuropathy ${ }^{[12]}$; infection, tingling numbness, headache, hirsutism, gingival hyperplasia ${ }^{[20]}$.

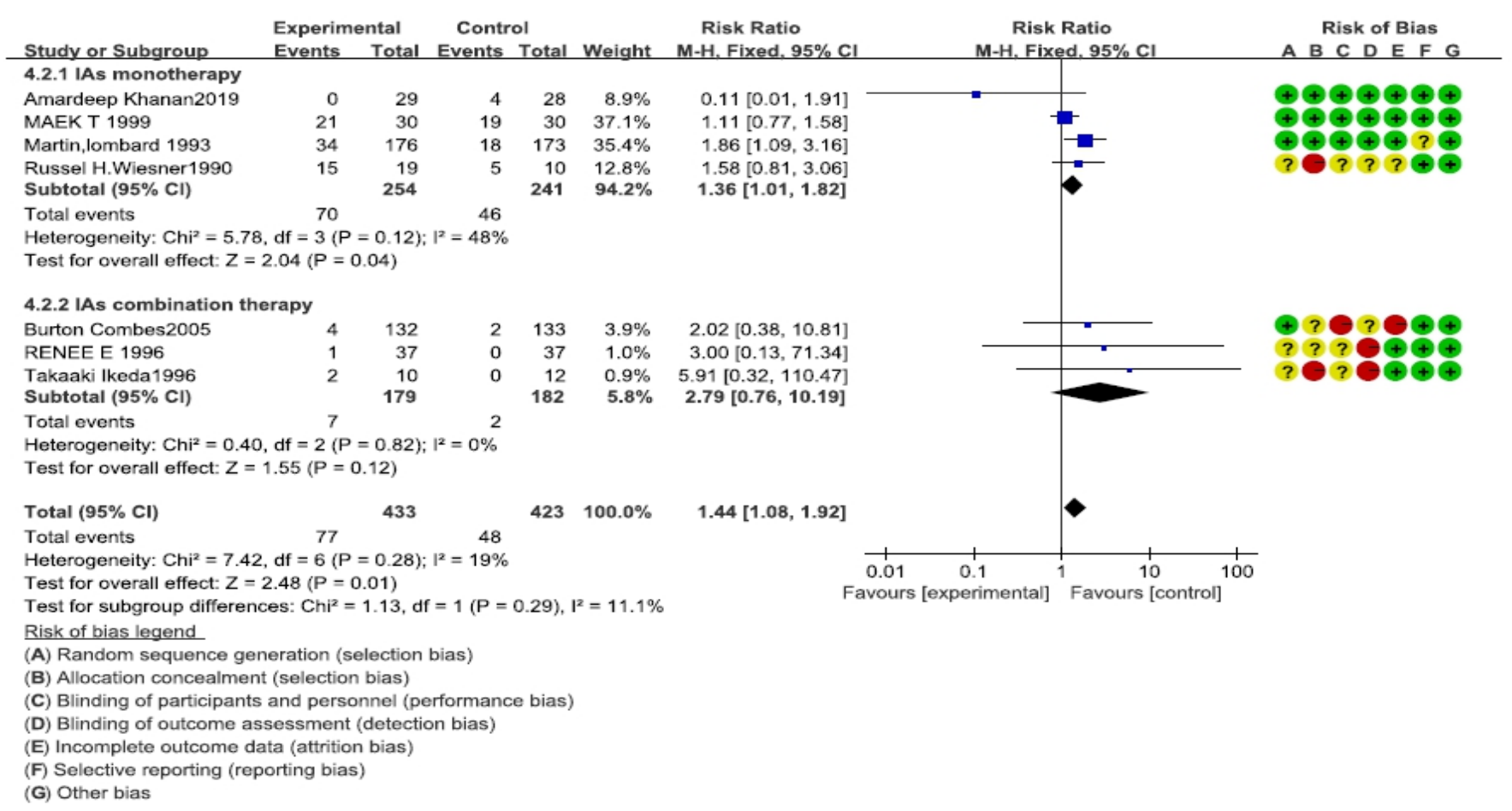

Figure 4. Forest plot of adverse effects

\subsection{Liver biochemical indicators}

This study also evaluated the efficacy of IAs therapy in improving liver biochemical indexes (ALP, ALT, IgM, bilirubin) in PBC patients. Table 2. showed that IAs therapy had no significant improvement on liver biochemistry except ALP (WMD: -0.62 to -0.14$], P=0.002$ ), although compared with the control groups, it was slightly improved.

Table 2. WMD of biochemical variables in PBC patients

\begin{tabular}{llllll}
\hline & Studies & Participants & WMD & $95 \% \mathrm{CI}$ & $P$ \\
\hline ALP & 3 & 114 & -0.38 & {$[-0.62,-0.14]$} & $P=0.002$ \\
ALT & 3 & 114 & -0.37 & {$[-0.90 .0 .17]$} & $P=0.18$ \\
IgM & 3 & 114 & -0.17 & {$[0.48,0.24]$} & $P=0.27$ \\
Bilirubin & 2 & 94 & -0.85 & {$[-2.51 .0 .80]$} & $P=0.31$ \\
\hline
\end{tabular}




\section{Discussion}

Primary biliary cholangitis is characterized by progressive destruction of the small intrahepatic bile ducts, which can eventually progress to cholestasis, advanced fibrosis, cirrhosis and liver failure. In recent years, multi-country researchers investigated the therapeutic effect of IAs (such as colchicine, cyclosporine, methotrexate, azathioprine, budesonide, rituximab, etc.) for $\mathrm{PBC}$ from the perspective of immunosuppressive agents. However, the clinical benefit and risk associated with the administration of IAs in PBC patients remain inconclusive. Therefore, this meta-analysis aimed to evaluate the potential therapeutic value and safety of IAs in primary biliary cholangitis.

A recent network meta-analysis ${ }^{[21]}$ reviewed the efficacy and safety of comprehensive therapies (including UDCA, corticosteroids, bezafibrate, colchicine, azathioprine, etc.) in patients with PBC, founding that none of the 12 treatments had a statistically significant reduction in mortality or liver transplantation in PBC patients, and compared with UDCA, monotherapy with colchicine, cyclosporin, methotrexate were associated with statistically significant increased risk of adverse effects. These findings are consistent with our results. Response of ALP to therapy has been shown to be a good correlate of both survival and liver histology in PBC and is used globally in clinical practice to predict the progression of the disease ${ }^{[4]}$. Compared with the control groups, IAs significantly reduced the serum alkaline phosphatases level of $\mathrm{PBC}$ patients, indicating that it is potential to treat $\mathrm{PBC}$.

The pathological progression of $\mathrm{PBC}$ can be divided into four clinical stages, the pre-clinical stage is marked solely by a positive anti-mitochondrial antibody without symptoms or abnormal liver biochemical tests ${ }^{[22]}$. The asymptomatic stage is characterized with the development of abnormal liver tests. At the stage of disease progression, the most common clinical symptoms including pruritus, fatigue, excessive skin pigmentation, liver and spleen enlargement, and xanthoma affect the quality of life. The advanced stage of PBC is marked by the development of cirrhosis and liver failure, requiring liver transplantation. Without treatment, the median survival for symptomatic patients is 6-10 years, with an accelerated course after development of ascites and hepatic encephalopathy ${ }^{[4]}$. At present, the only approved medical treatment for PBC targets bile acid homeostasis, and UDCA remains the only first-line treatment drug [23]. Although UDCA can slow down pathological progression, it seems to be ineffective in treating clinical symptoms, such as pruritus and fatigue ${ }^{[7]}$. Also, $40 \%$ of patients have incomplete response to UDCA. A number of pharmaceutical preparations have been explored in recent years as adjunct therapy for PBC patients who have incomplete response to UDCA. A retrospective study reported a randomized controlled clinical trial of MTX and colchicine combined with UDCA for PBC patients, with 29 patients most of whom observed to have significant clinical improvement after 20 years ${ }^{[24]}$. Of the eight patients who survived in the MTX combined UDCA group, seven patients had no signs of portal hypertension on liver biopsy, and one patient with esophageal varices did not deteriorate after 19 more years of follow-up, suggesting that MTX combined with UDCA may have potential therapeutic value in patients with incomplete UDCA response. Budesonide is a nonhalogenated glucocorticoid absorbed in the small intestine, prospective controlled studies found that budesonide improved liver biochemical enzymes and immune indicators in patients with incomplete UDCA responses, as well as improved histological scores ${ }^{[25]}$. However, these studies did not report outcomes for mortality or liver transplantation rates, and Budesonide appears to be associated with a significant worsening of osteoporosis in PBC patients [26]. In addition, immune-modulating medications such as azathioprine and mycophenolate mofetil [27] have been explored with unsatisfactory results.

With the development of immune technology, biological agents with more potential to treat $\mathrm{PBC}$ were found, such as Ustekinumab, Abatacept, CD40/CD40L Blockade, rituximab, etc. [28] . Anti-mitochondrial antibodies targeting pyruvate dehydrogenase can cause fatigue in PBC patients by causing muscle aerobic metabolic disorder and reducing exercise tolerance, while rituximab can reduce 
PBC antibodies by consuming B cells, thus improving fatigue ${ }^{[29]}$. However, there was no evidence that Rituximab had a 
significant effect in the treatment of PBC fatigue. The efficacy of immunosuppressive agents in PBC seems pessimistic, and further clinical studies on the IAs therapy may be need to prove it.

\subsection{Conclusion}

IAs cannot reduce the risk of mortality or liver transplantation, whether in IAs monotherapy or combination therapy, and even be associated with more adverse effects. However, IAs treatment can markedly reduce the serum levels of ALP in PBC patients, indicating that IAs still have therapeutic potential.

This study has some limitations: only RCTs included in our study, without cohort and case-control studies, the sample size was small. Secondly, there were insufficient data to record changes in the histological parameters, the quality of life and clinical symptoms, only one research reported changes in pruritus and fatigue score and the smaller trials were less statistically significant.

\section{Disclosure statement}

The author declares no conflict of interest.

\section{References}

[1] Gulamhusein AF, Hirschfield GM., 2020, PBC: Pathogenesis and Therapeutic Opportunities. Nature Reviews. Gastroenterology \& Hepatology, 17(2):93-110.

[2] Selmi C, Lleo A, Pasini S, et al., 2009, Innate Immunity and PBC. Current Molecular Medicine, 9(1):45-51.

[3] Carey EJ, Ali AH, Lindor KD., 2015, PBC. The Lancet, 386(10003):1565-1575.

[4] Silveira MG, Brunt EM, Heathcote J, et al., 2010, American Association for the Study of Liver Diseases Endpoints Conference: Design and Endpoints for Clinical Trials in PBC. Hepatology (Baltimore, Md.), 52(1):349-359.

[5] Poupon RE, Lindor KD, Cauch-Dudek K, et al., 1997, Combined Analysis of Randomized Controlled Trials of UDCA in PBC. Gastroenterology, 113(3):884-890.

[6] Parés A, Caballería L, Rodés J, et al., 2000, Long-Term Effects of UDCA in PBC: Results of a Double-Blind Controlled Multicentric Trial. Journal of Hepatology, 32(4):561-566.

[7] Beuers U, Lindor KD., 2011, A Major Step Towards Effective Treatment Evaluation in PBC. Journal of Hepatology, 55(6):1178-1180.

[8] Azemoto N, Abe M, Murata Y, et al., 2009, Early Biochemical Response to UDCA Predicts Symptom Development in Patients with Asymptomatic PBC. Journal of Gastroenterology, 44(6):630-634.

[9] Pascart T, Richette P., 2018, Colchicine in Gout: An Update. Current Pharmaceutical Design, 24(6):684-689.

[10] Mika A, Stepnowski P., 2016, Current Methods of the Analysis of IAs in Clinical Materials: A Review. Journal of Pharmaceutical and Biomedical Analysis, 127:207-231.

[11] QIAN J-D, et al., 2000, Treatment of PBC with UDCA, Prednisolone and Immunosuppressants in Patients Not Responding to UDCA Alone and the Prognostic Indicators. Clinics and Research in Hepatology and Gastroenterology. DOI: https://doi.org/10.1016/j.clinre.2020.03.026.

[12] Poupon RE, Huet PM, Poupon R, et al., 1996, Randomized Trial Comparing Colchicine and UDCA Combination to UDCA in PBC. Hepatology. 24(5):1098-1103.

[13] Almasio LL, Floreani A, Chiaramonte M, et al., 2000, Multicentre Randomized Placebo-Controlled Trial of UDCA With or Without Colchicine in Symptomatic PBC. Aliment 
Pharmacol Ther. 14(12):1645-1652. 
[14] Wolfhagen FH, VAN Hoogstraten HJ, Van Buuren HR, et al., 1998, Triple Therapy with UDCA, Prednisone and Azathioprine in PBC: a 1-Year Randomized, Placebo-Controlled Study. J Hepatol., 29(5):736-742.

[15] Combes B, Emerson SS, Flye NL, et al., 2005, Methotrexate (MTX) Plus UDCA in the Treatment of PBC. Hepatology (Baltimore, Md.), 42(5):1184-1193.

[16] Hendrickse MT, Rigney E, Giaffer MH, et al., 1999, Low-Dose Methotrexate is Ineffective in PBC: Long-Term Results of a Placebo-Controlled Trial. Gastroenterology, 117(2):400-407.

[17] Ikeda T, Tozuka S, Noguchi O, et al., 1996, Effects of Additional Administration of Colchicine in UDCA-Treated Patients with PBC: A Prospective Randomized Study. Journal of Hepatology, 24(1):88-94.

[18] Khanna A, Jopson L, Howel D, et al., 2019, Rituximab Is Ineffective for Treatment of Fatigue in Primary Biliary Cholangitis: A Phase 2 Randomized Controlled Trial. Hepatology, 70(5):1646-1657.

[19] Lombard M, Portmann B, Neuberger J, et al., 1993, Cyclosporin a Treatment in PBC: Results of a Long-Term Placebo-Controlled Trial. Gastroenterology, 104(2):519-526. DOI: 10.1016/0016-5085(93)90422-9.

[20] Wiesner RH, Ludwig J, Lindor KD, et al., 1990, A Controlled Trial of Cyclosporine in the Treatment of Primary Biliary Cirrhosis. N Engl J Med, 322(20):1419-24.

[21] Zhu GQ, Huang S, Huang GQ, et al., 2015, Optimal Drug Regimens for PBC: A Systematic Review and Network Meta-Analysis. Oncotarget, 6(27):24533-24549.

[22] Suraweera D, Rahal H, Jimenez M, et al., 2017, Treatment of PBC UDCA non-responders: A Systematic Review. Liver International: Official Journal of the International Association for the Study of the Liver, 37(12):1877-1886. DOI: 10.1111/liv.13477.

[23] Shah RA, Kowdley KV., 2020, Current and Potential Treatments for PBC. The Lancet Gastroenterology \& Hepatology, 5(3):306-315. DOI: 10.1016/S2468-1253(19)30343-7.

[24] Leung J, Bonis PA, Kaplan MM., 2011, Colchicine or Methotrexate, with Ursodiol, are Effective After 20 Years in a Subset of Patients with Primary Biliary Cirrhosis. Clinical Gastroenterology and Hepatology: The Official Clinical Practice Journal of the American Gastroenterological Association, 9(9):776-780.

[25] Rautiainen H, Kärkkäinen P, Karvonen AL, et al., 2005, Budesonide Combined with UDCA to Improve Liver Histology in PBC: A Three-Year Randomized Trial. Hepatology (Baltimore, Md.), 41(4):747-752.

[26] Rautiainen H, Färkkilä M, Neuvonen M, et al., 2006, Pharmacokinetics and Bone Effects of Budesonide in PBC. Alimentary Pharmacology \& Therapeutics, 24(11-12):1545-1552.

[27] Treiber G, Malfertheiner P., 2005, Mycophenolate Mofetil for the Treatment of PBC in Patients with an Incomplete Response to Ursodeoxycholic Acid. Journal of Clinical Gastroenterology, 39(9):837-8.

[28] Bowlus CL, Yang GX, Liu CH, et al., 2019, Therapeutic Trials of Biologics in Primary Biliary Cholangitis: An Open Label Study of Abatacept and Review of the Literature. Journal of Autoimmunity, 101:26-34.

[29] Tsuda M, Moritoki Y, Lian ZX, et al., 2012, Biochemical and Immunologic Effects of Rituximab in Patients with Primary Biliary Cirrhosis and an Incomplete Response to Ursodeoxycholic Acid. Hepatology (Baltimore, Md.), 55(2):512-521. 